\title{
Atopic dermatitis in adolescents with acne vulgaris
}

Atopic dermatitis $(A D)$ is a chronic skin disease characterized by severe pruritus, erythema, edema, crusting, excoriation, lichenification and skin dryness. AD is oft... diagnosed during childhood, though the onset of symptoms may occur at any tim is Lesions in older children and adults are usually located in flexural areas along wi $\mathrm{h}$ tr.? head and neck. Facial involvement is also common in $A D$, not only in chilni $n$ wut additionally in adolescents [1]. This inflammatory disease is characterizer n.' skin microbial dysbiosis and barrier dysfunction. Initial inflammation has a $\mathbf{T} 2$ pr ufile in response to allergens, which is later amplified by skin barrier breaking ar a rexuction of antimicrobial peptides. This promotes skin pathogens growth (mainly siurnylococcus aureus) and the evolution to a T2, T1 and T17 pattern [2, 3]. AD is $c^{c_{+}}$en associated with other atopic diseases such as rhinitis and asthma.

Acne vulgaris (AV) is highly prevalent in adolescent and acco-din affects nearly all people between the ages of 15 and 17 yeirs ; 7]. This multifactorial disease is associated with changes in the microbiome, hormo, $\because$. status and sebaceous production that leads to a cutaneous inflammatory pror sss meaiated by T1 and T 17 cells. This process also involves the activation of TOLL 'in receptors by the antigens of Cutibacterium acnes [5]. Acne lesions are classifiec is $n^{n}{ }^{\prime}{ }_{\text {, }}$-inflammatory (comedones) or inflammatory (papules, pustules and nodules) ana icatricial acne. As for severity, acne is classified from minimal to very severe, - cu vrding to the extension and type of lesions. Disruption of the skin barrier in arr facilitates the entry of additional allergens or other antigens $[2,5]$.

Atopic dermatitis is treated with topical suids and topical immunosuppressants. In more severe cases of AD, systemic cortı nir', biologic drugs (anti interleukins or their receptors) and JAK inhibitors shou'd 0 : cunsidered [6]. Acne treatment relies on antibiotics, retinoids and sebum rivaution modifiers. In females, hormonal therapy may also be added [7]. Self-caro, 10 -sures can improve disease control and prevent new outbreaks in both conditio 's.

Both acne vulgaris and atop $=$ de matitis have a great impact on quality of life [8].

A recent study concludeu that individuals with $A D$ have the same prevalence of $A V$ as non-AD individuals $[\mathrm{H}]$. Considering the different pathogenesis of these clinical conditions and givel, the nigh morbidity associated with atopic dermatitis and acne in pediatric populatic. our aim was to investigate if adolescents with AV have different expressions of $A D$ ar $d$ the impact in quality of life of both diseases.

For this purf $\sim 2$, we selected a representative sample of people aged 14-17 years. A questionnair was distributed randomly to students from four different schools in Lisbon, Dri ;al, between February and June of 2021. Acne vulgaris was diagnosed in $\left.71.3 \%{ }^{195} \% \mathrm{Cl} 65.6-77.3\right)$ of the adolescents. A cross-sectional, prospective study inve iv' . $\tau$ the high school students with acne vulgaris $(n=170)$ was then performed. The $\therefore$ ost - . nnaire collected demographic data, clinical information about acne, atopic $d \epsilon$-matitis, asthma and rhinitis and treatments used. The cohort also responded to ?ermatology Life Quality Index (DLQI) and to Global Health Evaluation Scale (GHES). 
Statistical analysis was done using non-parametric tests for group comparison, as data did not meet the normality assumption. For categorical variables, we used Fisher's test and for continuous variables, Wilcoxon rank sum/Mann-Whitney test was used. There was statistical significance for $p$-values $<0.05$.

This study obtained ethical approval from the Ethics Committee of Hospital janiz Maria on $20^{\text {th }}$ of September 2020. All participants and respective parents $n_{1}$ w/lued informed consents.

The results are summarized in Table 1 and Table 2.

[Insert Table 1.]

[Insert Table 2.]

We found a frequency of $12.4 \%$ of atopic dermatitis in adolesct. 'ts with acne. In a recent international study, that involved adolescents aged 1) : 18 years from 18 countries, the following values of $A D$ prevalence were fou, $\downarrow$ ir European Countries: Germany 8.7\%; France 14.4\%; UK 15\%; Italy 18\% and S rain 12.8\% [10]. Few studies address skin diseases prevalence in this age group. Tho 1. ' quency of $A D$ in the acne cohort is in agreement with population-based stur'ies of a topic dermatitis prevalence, although closer to the lower values reported in more. 'sent studies.

Although the pathophysiological mechanism o' $a_{\imath}$ 'vic dermatitis could justify lower expression of the disease in patients with $x_{-2}$, the chronic inflammation and disruption of the barrier function could ind "e, in contrast, the development of AD. Acne or AD often triggers the onset of "sk1 - ricking" which worsens both conditions [11]. Accordingly, this study showed t' at $+D$ was present in adolescents with worse acne evolution, more scars and $\cdot n_{1}$ ? severe type of acne, despite similar demographic characteristics of both s, $h$-óroups $[2,3,5]$.

Diagnosis by physician was re'orteu by more than half of the patients with atopic dermatitis and only $39 \%$ of tho $r^{-t}$.ents with isolated acne. The use of treatments and adjuvants was similar in bot.: ', $r$ ups. In fact, $80 \%$ of the adolescents confirmed the use of cleaning gel, moisturi-ing creams, solar protector, exfoliator, make up or make-up remover irrespective of the presence of $A D$. This seems to be a positive finding, as emollients and other, nn- nedical products may decrease the number of flares and the use of medication. Av ila ility of these products increases long-term adherence [12]. Medical treatme -.t 1 .nged from 62 to $67 \%$, which is a relatively low percentage. The disease chronic ${ }^{+} v$, frequent relapses and reduced medical diagnosis could be a plausive expl $r_{\varkappa}$ ion to the reduced therapeutic accomplishment [13].

Adolesceri: $v$ 'ith AD used corticosteroids more often and the difference observed betwe $\leftarrow \mathrm{n}$ croups was statistically significant, as expected [6]. Interestingly, no diffe:-nas were observed concerning asthma/rhinitis diagnosis or the use of ntin st imines.

$\mathrm{C}_{\mathrm{i}, \mathrm{l}}$ ᄀlly, adolescents with both conditions had lower scores of Dermatology Life Quality 1. Hex (DLQI) and felt more embarrassed because of their skin appearance. This difference was statistically significant. The presence of lesions in visible areas of skin may cause emotional troubles in patients, including low self-worth, embarrassment, 
sorrow and social isolation. Mental disorders and affected quality of life is reported in patients with acne vulgaris, atopic dermatitis, psoriasis, ichthyosis, vitiligo, and hidradenitis suppurativa [8]. Although adolescent acne is mainly considered minimal or mild and atopic dermatitis is classified as mild by most of the adolescent patients 8 , $14]$, the chronic treatment requirements and relapsing courses may increase the perception of symptom severity and affect negatively the quality of lift $\left[\varepsilon_{\mathrm{J}}\right.$. Adolescence is a period of time with several biological, social and emotiona tha. and the burden of two diseases that have a great impact on the quality of 1 " ? snould be taken into consideration. To our knowledge, this is the first study tha+ $d{ }^{-1}=$ rmines $A D$ frequency among adolescents with acne and the impact of these dis sasf $s$ on their quality of life. These findings should be considered in the managem $n t$, $f$ adolescents with these skin diseases.

\section{Declarations:}

- Acknowledgements: There are no acknowledgements.

- Conflicts of Interest: The authors declare tha+ there were no conflicts of interest in conducting this work.

- Funding: There is no funding to declare.

\section{References:}

1. Girolomoni, G., et al., Nor:.nc' 'ture and clinical phenotypes of atopic dermatitis. Therapeutic Acran es in Chronic Disease, 2021. 12: p. 204062232110029.

2. Pistone, D., et al., A Jurney on the Skin Microbiome: Pitfalls and Opportunities. Internati nal Journal of Molecular Sciences, 2021. 22(18): p. 9846.

3. Kortekaas Krohn, I., $i_{i}$ al., T-cell subsets in the skin and their role in inflammatory ski, tisorders. Allergy, 2021.

4. Bhate, K. and 1.C Williams, Epidemiology of acne vulgaris. British Journal of Dermatology, 2 1? 168(3): p. 474-485.

5. Cong, T.-Y el al., From pathogenesis of acne vulgaris to anti-acne agents. Archives of Jurmatological Research, 2019. 311(5): p. 337-349.

6. Salvati L. L. Cosmi, and F. Annunziato, From Emollients to Biologicals: Targe ${ }^{*}$. .g Atopic Dermatitis. International Journal of Molecular Sciences, 2021. 22(1s; p. 10381.

7. Ogt, I.K., A. Broussard, and M.D. Marshall, Acne Vulgaris: Diagnosis and Treuiment. Am Fam Physician, 2019. 100(8): p. 475-484.

8. Curtés, H., et al., Alterations in mental health and quality of life in patients with kin disorders: a narrative review. Int J Dermatol, 2021.

9. Halling, A.S., et al., No association between atopic dermatitis and acne vulgaris in the general population. J Eur Acad Dermatol Venereol, 2021. 35(4): p. e276e278.

10. Silverberg, J.I., et al., Atopic dermatitis in the pediatric population. Annals of Allergy, Asthma \& Immunology, 2021. 126(4): p. 417-428.e2. 
11. Lochner, C., A. Roos, and D. Stein, Excoriation (skin-picking) disorder: a systematic review of treatment options. Neuropsychiatric Disease and Treatment, 2017. Volume 13: p. 1867-1872.

12. Lopes, A., et al., Atopic Dermatitis Host and Environment Model: Revisiti ig Therapeutic Options. European Annals of Allergy and Clinical Immunolnoy, 2020. 52(01): p. 4.

13. Habeshian, K.A. and B.A. Cohen, Current Issues in the Treatmen ${ }^{+}$oj $^{\prime}$ Acne Vulgaris. Pediatrics, 2020. 145(Supplement 2): p. S225-S230.

14. Ezzedine, K., et al., Impact of Atopic Dermatitis in Adolescent an it their Parents: A French Study. Acta Derm Venereol, 2020. 100(17): p. ‘dv0u 294. 